improvement within 1 week. By 3 months, he returned to his neuropsychological baseline in majority of cognitive domains from moderate-severe dysfunction, with concurrent MRI demonstrating resolving white matter lesions and FFA showing less evident vasculitis. The treatment response was maintained with tapering of steroids $(25 \mathrm{mg}$ at 12 months). $\mathrm{He}$ was able to return to his previous occupation as a paramedic by 1 year.

Conclusion SuS is a rare, immune-mediated microangiopathy in which early recognition with aggressive immunosuppression is required to achieve optimal outcome. No randomized controlled trial (RCT) exists for the management of this condition. In this report, early recognition through multidisciplinary input and aggressive immunotherapy with rituximab resulted in a favourable outcome. However, RCT evidence is needed to guide management.

\section{CLINICAL AND NEUROPHYSIOLOGICAL IMPROVEMENT IN HEREDITARY SENSORY AND AUTONOMIC NEUROPATHY TYPE I (HSAN-1) FOLLOWING HIGH DOSE SERINE THERAPY}

${ }^{1}$ James D Triplett, ${ }^{1,2,3}$ Garth Nicholson, ${ }^{1,2,4}$ Con Yiannikas. ${ }^{1}$ Department of Neurology and Neurophysiology, Concord Repatriation General Hospital, Sydney, Australia, Concord, NSW, Australia; ${ }^{2}$ Sydney Medical School, University of Sydney, Sydney, NSW, Australia; ${ }^{3}$ Northcott Neuroscience Laboratory, ANZAC Research Institute, Sydney, NSW, Australia; ${ }^{4}$ Department of Neurology, Royal North Shore Hospital, Sydney, NSW, Australia

\subsection{6/bmjno-2021-ANZAN.113}

Objective To report clinical stabilisation and improved summated compound motor action potentials (CMAP) in a patient with Hereditary sensory and autonomic neuropathy type I (HSAN-1) following high dose serine therapy.

Case A 52-year-old male presented in 2006 with a typical HSAN-1c phenotype and over the ensuing years had progressive distal to proximal sensory disturbance, associated lancinating pains, and mild progressive distal predominant limb weakness. A Ser384Phe mutation in the SPTLC2 gene located on chromosome $14 \mathrm{q} 24$ was identified in this patient in 2017. In late 2018 high dose serine therapy (11 grams TDS) was commenced, resulting in stabilisation of clinical weakness.

Lower limb motor and sensory responses were absent at presentation in 2006 with initial summated bilateral upperlimb median and ulnar nerve CMAP being $31.2 \mathrm{mV}$ and steadily declining to $4.2 \mathrm{mV}$ in March 2019. Following Serine therapy, the summated CMAP increased to $5.4 \mathrm{mV}$ in August 2020 and $9.5 \mathrm{mV}$ in February 2021, furthermore previously absent upper-limb sensory nerve responses are now recordable.

Conclusions High dose Serine replacement therapy may lead to clinical stabilisation and improved neurophysiological parameters in HSAN-1. HSAN-1, an autosomal dominant sensory neuropathy occurs secondary to mutations in the enzyme Serine-Palmitoyltransferase (SPT), an essential enzyme in the de-novo synthesis of sphingolipids. The administration of high dose Serine may overcome altered SPT substrate specificity in HSAN-1, which preferentially uses L-alanine and L-glycine instead of L-serine and allow the formation of typical 1-deoxysphingolipids as opposed to atypical 1-deoxysphingolipids generally seen in this condition, with early treatment possibly preventing clinical progression.

\section{4 MAN-IN-THE-BARREL SYNDROME AS A COMPLICATION OF CERVICAL DECOMPRESSIVE SURGERY}

Rajiv Wijesinghe, Con Yiannikas. Department of Neurology, Concord Repatriation General Hospital, Concord, NSW, Australia

\subsection{6/bmino-2021-ANZAN.114}

Objective To present a case of painful brachial diplegia following cervical decompressive surgery.

Case A 73 year-old male presented with a 6 month history tripping over his left leg, resulting in near falls. An MRI demonstrated severe spondylotic cervical canal stenosis at C4/5 with myelomalacia and he subsequently underwent a cervical decompression and fusion at this level. On post-operative day five he developed severe pain in his neck and shoulders and mild weakness of his left arm. One week later he underwent a second operation with decompression and rhizolysis at C5 to $\mathrm{C} 8$. On post-operative day 3 he awoke with severe pain similar to previously, followed a day later by profound weakness of all movements around the shoulders bilaterally, but movements around the elbows, wrists and fingers were normal. While walking his arms hung limply beside him, giving him the appearance of a man in a barrel. Routine nerve conduction studies and median somatosensory evoked potentials were normal. Eelectromyography confirmed denervation within the C5 myotome bilaterally, however with selective sparing of the rhomboids bilaterally. This suggested a lesion distal to the branch to rhomboids, and a diagnosis of bilateral post-operative brachial neuritis was made.

Conclusion Acute proximal arm weakness is an uncommon complication of cervical surgeries, referred to commonly as a post-operative C5 palsy. Investigations performed in this case however suggest that a brachial plexus lesion may be the cause of this peculiar syndrome. This syndrome may lie on the spectrum of post-surgical inflammatory neuropathies.

\section{ERRONEOUSLY NORMAL ACTIVE B12 LEVEL IN A CASE OF SUBACUTE COMBINED DEGENERATION OF THE CORD}

${ }^{1}$ Nicholas Halliwell, ${ }^{2}$ Cecily Forsyth, ${ }^{3}$ Nicholas Taylor, ${ }^{2}$ Sarah Mangalasseril, ${ }^{1} J o n a t h a n$ Sturm. 'Neurology, Gosford Hospital, Gosford, NSW, Australia; ${ }^{2}$ Haematology, Gosford Hospital, Gosford, NSW, Australia; ${ }^{3}$ Clinical Biochemistry, Douglass Hanly Moir Pathology, Wentworth, NSW, Australia

\subsection{6/bmjno-2021-ANZAN.115}

Objectives Vitamin B12 is crucial for neurologic function, red blood cell production, and DNA synthesis. Deficiency can lead to a wide spectrum of haematologic and neuropsychiatric disorders including subacute combined degeneration of the cord. This report presents a case of a 47 y.o female who presented with subacute combined degeneration of the spinal cord with a normal active B12 level.

Results MRI demonstrated high T2 signal throughout the dorsal columns of the cervical and upper thoracic cord without enhancement consistent with subacute combined degeneration of the cord. Her blood count revealed a mild macrocytic anaemia. Total vitamin $\mathrm{B} 12$ was $<80 \mathrm{pmol} / \mathrm{L}$ and active B12 was $>128 \mathrm{pmol} / \mathrm{L}$, confirmed on repeat testing. Functional vitamin B12 deficiency was confirmed by an elevated homocysteine level of $38.6 \mathrm{umol} / \mathrm{L}$ (reference range 4.4 to 13.6 umol/L) and elevated serum methylmalonic acid of 20.75 